\title{
Structural differences between C-terminal regions of tropomyosin isoforms
}

Tropomyosins are actin-binding regulatory proteins, which overlap end-to-end along the filament. High resolution structures of the overlap regions were determined for muscle and non-muscle tropomyosins, however conformations of the junction regions in complex with actin are unknown. In this work, orientation of the overlap on actin alone and on actin-myosin complex was evaluated by measuring FRET distances between a donor (AEDANS) attached to tropomyosin and an acceptor (DABMI) bound to actin's Cys374. Donor was attached to the Cys residue introduced by site-directed mutagenesis near the C-terminal half of the overlap. The recombinant alpha-tropomyosin isoforms used in this study - skeletal muscle skTM, nonmuscle TM2 and TM5a, and chimeric TM1b9a had various amino acid sequences of the $\mathrm{N}$ and C-termini involved in the end-to-end overlap. The donor-acceptor distances calculated for each isoform varied between $36.4 \AA$ and $48.1 \AA$ Rigor binding of myosin $\mathrm{S} 1$ increased the apparent FRET distances of skTM and TM2, but decreased the distances separating TM5a and TM1b9a from actin. The results show that isoform-specific sequences of the end-to-end overlaps determine orientations and dynamics of tropomyosin isoforms on actin. This can be important for specificity of tropomyosin in the regulation of actin filament diverse functions. 
Małgorzata Śliwińska, Joanna Moraczewska

1 Institute of Experimental Biology, Kazimierz Wielki University, Bydgoszcz, Poland

2 | Corresponding author: Joanna Moraczewska

3 Address: Chodkiewicz 30 str., 85-064 Bydgoszcz, Poland

4 | phone numberTel: +48523419289

5 | eE-mail: moraczjo@ukw.edu.pl 


\section{Introduction}

7 Tropomyosins are-, a family of two-chain coiled coil proteins,

8 "gate keepers", which control access of numerous actin-binding proteins to actin filaments

9 (Gunning et al. 2008). Tropomyosin (TM) binds cooperatively to actin and due to end-to-end

10 overlap between adjacent molecules it forms long chains along both sides of the filament.

11 Binding of TM to actin involves weak, but specific electrostatic interactions between periodic

12 actin-binding sites on TM's coiled-coil and residues exposed on actin subunits (Barua et al. 2011; Li et al. 2011). In this closed state, TM inhibits activation of actomyosin ATPase at low myosin concentrations (Lehrer \& Morris 1982). Strongly bound myosin heads (S1) cooperatively shift the filament into the open state, which is associated with an azimuthal shift of TM away from the position occupied in the closed state (Lehman \& Craig 2008). The S1-

17 induced shift from the closed to the open state is a universal mechanism of actin filament

18 activation executed in the presence of muscle and non-muscle TM isoforms.

$\mathrm{TM}$ isoforms are generated by several genes (four in vertebrates), selection of

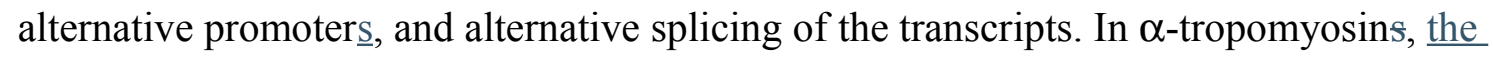
products of the TPMI gene, the N- and C-terminal regions are encoded respectively by two (1a and $1 b)$ and four (9a-d) alternative exons. Selection of the alternative promoter gives rise

23 to high molecular weight (HMW) and low molecular weight (LMW) isoforms of TM. HMW tropomyosins bind along seven actin subunits, whereas LMW isoforms along six actin

25 subunits. The main structural difference between these two TM types is the N-terminal sequence, which is encoded by exon 1a or $1 \mathrm{~b}$ respeetively in HMW and LMW isoforms, 27 respectively (Lees-Miller \& Helfman 1991). 
Structures of tropomyosin intermolecular junctions were studied with the use of model

29 peptides imitating sequences of the end-to-end overlaps. Peptides with sequences of skeletal

30 TM encoded by exons 1a and 9a (Greenfield et al. 2006; Murakami et al. 2008), non-muscle

31 TM encoded by exons $1 \mathrm{~b}$ and $9 \mathrm{~d}$ (Greenfield et al. 2009), and smooth muscle TM encoded by

32 exons 1a and 9d (Frye et al. 2010) were analyzed to obtain NMR and X-ray structures.

33 Although the structures differ in the number of amino acids forming the overlap and in

34 specific interactions between amino acid chains, the three complexes are similar $=$ the two $\alpha$ -

35 helical chains of the C-terminus spread apart and interlock with the N-terminal coiled coils.

36 The structures revealed, however, a slightly different tilt of the axis of the C-and N-terminal

37 coiled coil (Frye et al. 2010).

38 For understanding of the molecular mechanisms controlling numerous functions of actin, high--resolution structures of F-actin in complex with different TM isoforms are required. Models of actin complexes with skeletal and cardiac muscle TMs in different activation states are available (Barua et al. 2013; Barua et al. 2011; Barua et al. 2012;

43 between conserved residues repeting along TM and charged or hydrophobic residues exposed on actin (Barua et al. 2011; Li et al. 2011). When bound to actin alone, the N-terminal residues Lys6 and Gln9, located with the overlap region, make important interactions with Asp25 on actin. On the other hand, the C-terminal half of the overlap does not contribute directly to actin binding. This region eotlt may be important for regulation of myosin interactions with the filament, hererere the is no experimental data supporting this hypothesis. determine apparent distances between donors specifically attached to the N-terminal regions of different TM isoforms. Our data suggested that ${ }_{2}$ in closed and myosin-induced open states the N- 
52 terminal segments of tropomyosin isoforms are differently oriented on F-actin (Sliwinska et al.

53 2011). However, the results did not provide any insight into the erientationposition of C-terminal

54 segments of the studied isoforms. We do not know whether the regions located in the close

55 vicinity to the ofend-to-end junctions are stiff or rather flexible. Various flexibilities within this

56 region of TM might be an important determinant of differences between TM isoforms in

57 regulation of actin-myosin interactions.

58 The aim of this study was to analyze the erientation-positions of the C-terminal regions,

59 adjacent to the end-to-end overlap, in four tropomyosin isoforms. Steady-state FRET between $\underline{\mathrm{a}}$

60 donor attached to the Cys residue introduced into the C-terminal segment of each isoform and

61 acceptor bound to actin's penultimate Cys374 was used. The data shows that erientation

62 localization of the C-terminal region in relation to actin's C-terminus is unique for each type of

63 the studied isoform. Specific conformational changes associated with activation of the filament

64 by strongly bound myosin heads suggest that depending on the sequence, the end-to-end overlap

65 regions have different flexibilities.

66 Materials and Methods

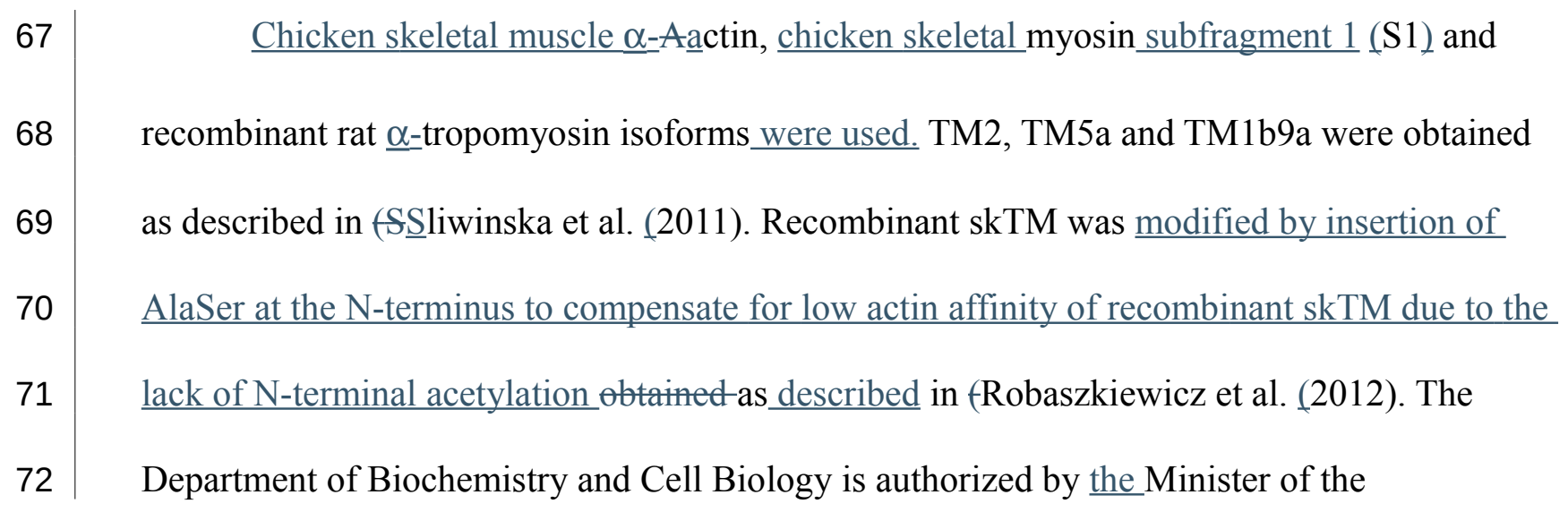


73 Environment (Poland) for laboratory use of genetically modified organisms (permit nr GMO:

$74 \quad 01-112 / 112)$.

75 PCR-based oligonucleotide-directed mutagenesis (Stratagene) was used to create an

76 attachment site for a fluorescent probe at the C-terminal region of TM. First, cDNA encoding

77 all TM isoforms used in this study was mutated to replace the single Cys codon for Ser. The

78 procedure was described in (Sliwinska et al. (2011). Thean cDNA from the first stage of

79 mutagenesis was used to create Ala269Cys-skTM, Ser269Cys-TM2, Ala232Cys-TM1b9a and

80 Ser232Cys-TM5a mutants. The oligonucleotides used at this stage were the following:

81 9a Ala269(232)Cys: 5'-ctgaagtacaagtgcatcagcgaggagctggaccacg-3'

82 9d Ser269(232)Cys: 5'-gccaaagaagaaaacctttgcatgcaccagatgctggac-3'

83 All primers were synthesized and HPLC purified by the Laboratory of DNA Sequencing and

84 Oligonucleotide Synthesis, Institute of Biochemistry and Biophysics, Polish Academy of

85 Science (Warsaw, Poland).

86 Tropomyosin mutants were labeled with AEDANS and actin was labeled with DABMI

87 according to the procedure described before-previously (Sliwinska et al. 2011).

88 Tropomyosin affinity for actin was measured in a co-sedimentation assay. Increasing

89 amounts of tropomyosin (final concentrations varied between 0 and $10 \mu \mathrm{M}$ ) were mixed with

$90 \quad \operatorname{actin}(5 \mu \mathrm{M})$ in F-buffer: $2 \mathrm{mM}$ HEPES, pH 7.6, $40 \mathrm{mM} \mathrm{NaCl}, 5 \mathrm{mM} \mathrm{MgCl}_{2}$ at $22{ }^{\circ} \mathrm{C}$ and ultracentrifuged.

91 Protein content in supernatants and pellets was analyzed electrophoretically on SDS-PAGE as described in

92 (Skorzewski et al. 2009).

93 The activity of actomyosin ATPase was measured in F-buffer. Myosin S1 concentration

94 was at $0.8 \mu \mathrm{M}, \mathrm{F}$-actin was $9.2 \mu \mathrm{M}_{2}$ and tropomyosin was $1.2 \mu \mathrm{M}$. The reaction was started by 


\begin{abstract}
95 addition of MgATP to $5 \mathrm{mM}$ and stopped after $10 \mathrm{~min}$. with 3.3\% SDS and $30 \mathrm{mM}$ EDTA.
96 The amount of liberated phosphate was measured colorimetrically according to the method 97 described in (-White (1982).
98 Fluorescence anisotropy, FRET experiments, calculation of Förster critical distance $99\left(\mathrm{R}_{0}\right)_{2}$ and donor-acceptor distance $(\mathrm{R})$ were conducted according to the methods described in $100 \quad$ (Sliwinska et al. (2011).

\title{
$101 \quad$ Results
}

102 Rationale for the labeling-_site selection and characterization of the labeled tropomyosin 103 isoforms

End-to-end overlap sequences present in the studied TM isoforms were formed by two $\mathrm{N}$-terminal and two C-terminal variants of alternative sequences of rat $\alpha \mathrm{TM}$. $\underline{\mathrm{A}} \mathrm{S} \underline{\mathrm{s}} \mathrm{chematic}$

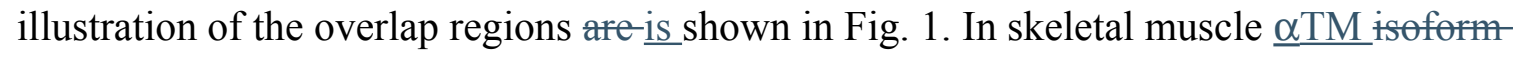
(skTM), non-muscle isoforms (TM2, TM5a), and in chimeric TM1b9a, an isoform an isoform whichthat has no natural counterpart, $\mathrm{N}$-termini encoded by exons $1 \mathrm{a}$ or $1 \mathrm{~b}$ formed complexes with C-termini encoded by exons $9 \mathrm{a}$ or $9 \mathrm{~d}$. In order to create a specific reactive site for attachment of a fluorescent probe to the C-terminal regions of tropomyosin isoforms, residue 269 in HMW isoforms or its counterpart in LMW isoforms (residue 232)

113 the outskirts of the end-to-end junction (Frye et al. 2010; Greenfield et al. 2006; Greenfield et for to Cys (Table. 1). This residue was selected for the following reasons: (a) it is located at al. 2009),, ; (b) it is not involved in any interactions within the studied end-to-end complexes (Frye et al. 2010); and (c) it is located on the outer surface of the two-chain coiled-coil 
tropomyosin (position $c$ of the coiled-coil heptapeptide repeat), which allows for free motions of the attached label, (d) it is part of the flexible region, where coiled coil strueture turns intoparallel heliees (Frye et al. 2010; Greenfield et al. 2003). Thus we expected that the attached

119 label was sensitive to conformational differences within the end-to-end overlap.

Replacement of Cys for Ser in the central region of TM did not affect the basic

121 functions such as actin binding and regulation of actomyosin ATPase activity (Sliwinska et al.

122 2011). In order to check whether these functions were conserved in TMs with Cys mutations in the $\mathrm{C}$-terminal region, we measured actin binding regulatory functions of all studied AEDANS-labeled TM isoforms. Binding constants $\left(\mathrm{K}_{\mathrm{app}}\right)$ obtained in the co-sedimentation assay show that all TM mutants bound to actin with high affinity (Table 2), consistent with previous work (Moraczewska et al., 1999). The mutations also did not ehange-significantly change the interactions between actin and myosin heads. In the presence of wild--type and mutant TMs the activities of actin-myosin S1 ATPase were similar (Table 2).

To check whether all isoforms of AEDANS-labeled TM bound to DABMI-labeled 130 actin stoichiometrically, TMs were titrated with increasing concentrations of actin. The titration curves shown in Fig. 2 indicate that the fluorescence was maximally quenched at the ratio of TM to F-actin, thieht that was close to stoichiometric. For skTM and TM2 the maximal quenching was reached at 1 TM to 6-7 actins and in the case of TM5a and TM1b9a at $1 \mathrm{TM}$ to 4-5 actins. The results confirm high actin affinity of TM isoforms labeled in the $\mathrm{C}$ terminal segment. Additionally, the titration curves show that in case of the isoforms with the

139 suggest that the C-terminal sequence encoded by exon $9 \mathrm{~d}$ facilitates interactions of the C140 terminal regions of TM2 and TM5a with actin. 
Distances separating the donor attached to the C-terminal region of tropomyosin from the acceptor bound to actin

Random orientation of the labels is important for FRET distance measurements

144 (Lakowicz 1999). To ensure that the probe was flexibly attached to Cys269/232 in all studied

145 TM isoforms, we measured AEDANS fluorescence anisotropies. As shown in Table 3, the obtained anisotropies were low, and therefore we concluded that the label bound to free TM as

147 well as to TM in complex with actin and with myosin S1 was randomly oriented. Thus, the

148 orientation of the donor did not limit the FRET distance measurements. and absorption of the acceptor $(\mathrm{J})$ affect donor-acceptor critical distance $\left(\mathrm{R}_{0}\right)$ (Lakowicz 1999). Because the local environment surrounding AEDANS bound to Cys269/232 could influence both parameters, $Q_{D}$ and $J$ were determined for each labeled TM mutant in the presence of unlabeled actin. The results show that the label attached to the C-terminal segment of the 154 studied TM isoforms was exposed to different environments, which caused variations in $Q_{D}$,

155 small shifts in $\mathrm{J}$ and, in consequence, differences in $\mathrm{R}_{0}$ (Table 4).

157 labeled TM in the absence and in the presence of acceptor (TM saturated with DABMI-F158 actin). The fluorescence of the donor was corrected for the increase caused by binding of 159 unlabeled actin (3-7\% depending on TM isoform). The efficiencies obtained for the studied

160 TM isoforms were used for calculations of the apparent distances $(\mathrm{R})$ separating donor and

161 acceptor. All calculated FRET parameters are collected in Table 4. The results suggest

162 isoform-specific localization of the overlap region. The differences between the distances 163 obtained for HMW isoforms (skTM, TM2) were small, which indicates similar position of the 
164 donor in relation to the acceptor. In LMW isoforms (TM5a and TM1b9a) the donor-acceptor

165 distances were larger. It is worth noting that the distance obtained for TM1b9a was far from

$166 \mathrm{R}_{0}$, thus the sensitivity of FRET measurements was limited and the result can only be regarded

167 as a rough estimate.

168 Changes in tropomyosins' C-termini as an effect of myosin S1 binding to actin

Strong binding of myosin heads (S1) to the filament increases affinity of TM to actin and induces an azimuthal shift of TM chains. This_changes the TM interactions with actin and

171 activates the filament allowing for actin-myosin cross-bridge cycling (Lehman \& Craig 2008;

172 Moraczewska 2002). Saturation of the filament with myosin S1 caused a significant increase

173 of the fluorescence of AEDANS bound to C-terminal segments of all studied TM isoforms.

174 The average increase was about $14 \%$ and $18 \%$ for skTM and the non-muscle isoforms,

175 respectively. Since the fluorescence intensity of the probe attached to the N-terminal segment

176 increased by about 2-9\% (Sliwinska et al. 2011), it appeared that the fluorophore bound to the

177 C-terminal segment of TM was more sensitive to myosin binding. The change of fluorescence

178 observed in this work suggested that the C-terminal region of TM either directly interacted

179 with myosin or significantly changed conformation upon myosin binding to actin. To explore

180 the later possibility, changes in FRET distances in the presence of myosin were analyzed.

181 Strongly bound S1-shifted the C-terminal regions of all TM isoforms, however but the

182 direction of the shift observed for HMW and LMW isoforms was different. As compared to the TM-actin complex, in the presence of S1 the energy transfer efficiency between AEDANS attached to skTM or TM2 and DABMI-actin decreased. In contrast, when the energy donor was attached to TM5a or TM1b9a, an increase in transfer efficiency was observed (Table 5). Binding of unlabeled actin-S1 did not shift the maximum of the fluorescence spectrum, thus 
187

the spectral overlap (J) was unchanged. However, the quantum yield of AEDANS-TM bound to unlabeled actin-S1 increased (Table 5), which. This called for recalculation of the critical distances (see equation 5 in (-Sliwinska et al. (2011)). Based on the new values of $\mathrm{R}_{0}$ and transfer efficiencies (E) obtained for AEDANS-TMs saturated with DABMI-actin-S1, donoracceptor distances were calculated (Table 5).

The degree of the maximal shift induced by $\mathrm{S} 1$ binding to actin $\left(\Delta \mathrm{R}_{\mathrm{S} 1}\right)$ was calculated as the difference between the donor-acceptor distance in the absence and in the presence of $\mathrm{S} 1$. The data indicates that the C-terminal region of the HMW isoforms was shifted away from the donor, whereas in LMW it was shifted closer to the donor.

The S1-induced activation of the filament is a very cooperative process, which. It means that the final change in TM orientation is achieved at S1 concentrations menterar below the concentrations required for actin saturation (Eaton 1976; Moraczewska 2002). Fig. 3 shows the effects of increasing S1 concentrations on energy transfer between donor-labeled TM and acceptor-labeled actin. The experimental data was normalized and fit to the Hill equation. The ratios of strongly bound myosin heads to actin required for the half-maximal change in the energy transfer (S1/actin molar ratio) are elleeted shown in Table 5. The numbers in parentheses show the S1/TM molar ratio, obtained by multiplying S1/actin by the number of actin subunits bound by one TM molecule. The data shows that the cooperativity was very high for each of the isoforms. The differences depended on the type of end-toend overlap.

\section{Discussion}

The position tropomyosin assumes on the filament controls interactions of the filament with many actin-binding proteins, thus it is an important determinant of actin filament functions. The present work is a continuation of our previous studies on the structural diversity among 
210 tropomyosin isoforms determined by sequences of the end-to-end junctions (Sliwinska et al.

211 2011). Here-In this study, erientations-positions of the C-terminal segments of four tropomyosin

212 isoforms relative to the actin C-terminal region were analyzed with the use of steady-state

213 FRET. Distances were measured between a donor specifically attached to the C-terminal segment

214 of TM and an acceptor bound to actin's penultimate Cys374, which is located in the outer domain

215 of the filament.

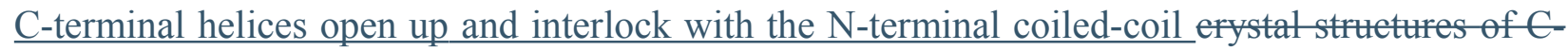
terminal segments of musele TMs, the C-terminal heliees open upen formation of the end-to-end eomplex (Greenfield et al. 2006; Greenfield et al., 2009; Frye et al. 2010). Although the high resolution structure of tropomyosin overlap bound to actin is not known, most probably this mode of tropomyosin end-to-end interaction along the actin filament is maintained, as it fits well into atomic models of F-actin-TM (Barua et al., 2011; Li et al., 2011). The results obtained in this work suggest that, when bound to actin, the overlap complex remains flexiblerealed that the orientation of $\mathrm{C}$-terminal segments of the studied TM isoforms was not determined by exon 9 eneoded sequenee, but rather by the sequenee of the end-to-end overtap complex. The donor- 
234

235

236

237

238

9a (skTM and TM1b9a) or 9d (TM2 and TM5a) differed by about 6-10. . This stggests that interdigitation of the C-terminal sequences with different types of $\mathrm{N}$-termini changes conformation of the C-terminal helices with by various degrees. The position of each C-terminal segment of the studied TM isoforms was not determined by the exon 9-encoded sequence, but rather by the sequence of the end-to-end overlap complex.

__ Interestingly, in the case of TM1b9a, which shares the same C-terminal sequenee with skTM (eneded by exon 9a), the donor was separated from the acceptor aetin by a much larger distance than in the three other isoforms indicating. This shows that the non-muscle $1 \mathrm{~b}$ encoded $\mathrm{N}$-terminus strongly distorts the structure of the striated muscle-specific $9 \mathrm{a}$-encoded C-terminus, , which is specifie for skeletal musele TM and forms complex with the 1a-eneoded $\mathrm{N}$-terminus.

In the earlier study ${ }_{2}$ wsedthree out of the four TMs used in this work were studied: TM2, TM5a-, and TM1b9a. The TMs were labeled with AEDANS in the $\mathrm{N}$-terminal segment (residues 23 or 28) to measure the distance separating the donor located near the $\mathrm{N}$-terminal half of the end-to-end junction and the acceptor bound to actin's (Cys374) (Sliwinska et al. 2011). In TM2, the FRET distances obtained for donors attached to the N-terminal and the C-terminal regions were 40.2 and $36.4 \AA$, respectively. In the case of TM5a, the respective distances were 39.3 and $42.7 \AA$. Taking into account the length of the donor and acceptor probes (about $10 \AA$ ) and their random distribution, the differences of about $3.5 \AA$ between both distances in the two isoforms were small. Thus, when bound to actin, TM2 and TM5a seem to be slightly bent within the end-to-end junction. In contrast, in TM1b9a the FRET distances measured from the $\mathrm{N}$ - and $\mathrm{C}$ terminus were 34.8 and $48.1 \AA$ respectively. The $13.3 \AA$ difference suggests that the overlap of this isoform is bent or even broken. The earteon shown in Fig. 4 compares the FRET distanees ebtained in this and the previous work. To localize the ends on the surface of actin monomer, we positioned the $\mathrm{N}$-terminus of $\mathrm{TM} 2$, eneoded by exon $1 \mathrm{a}$, over a path of aetin amino aeid residues 
D25, K326 and K328, which, according to the models of TM-actin, directly interact with TM's K6, E16, and D20 (Barta et al. 2013; Barta et al. 2011; Li et al. 2011). Since the C-terminal

261 residues of skeletal TM do not seem to eontribute to the TM-aetin interfaee (Bartu et al. 2013; Barta et al. 2011; Li et al. 2011), this region was positioned arbitrarily, to illustrate the apparent distanee separating Cys269 from aetin. Atomie models of aetin in eomplex with LMW TMs are not available, thus our FRET distances were used to position the ends of TM5a and TM1b9a relative to the position of TM2. Differences in FRET distances measured from $\mathrm{C}$-and $\mathrm{N}$-termini of TM2 and TM5a were $3.8 \AA$ and $3.4 \AA$, respectively, therefore it appears that in complex with aetin the $\mathrm{N}$ - and- $\mathrm{C}$-terminal regions of these wo isoforms were slightly bent. The differenee between donor-aeeeptor distanees measured from C-and N-termini of TMHb9a was about 13.3 $\AA$, whieh suggested that the end-to-end junetion of this isoform was either strongly bent or even broken. Significant curvature of the end-to-end overlap was also observed in X-ray and NMR structures of muscle and non-muscle TMs (Frye et al. 2010; Greenfield et al. 2009; Murakami et al. 2008). Together the results suggest that curvature of the overlap region is an inherent attribute of altTM isoforms. As suggested before, bending is important to adopt the helical structure of the

274 actin filament (Holmes \& Lehman 2008).

275 Our reeent studies have shown that dDuring the activation of the filament by strongly

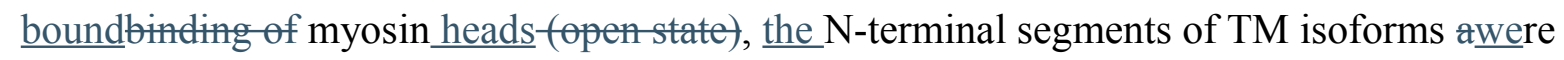


284

285

286

287

288

289

290

291

292

293

294

295

296

297

298

299

300

301

302

303

304

305

306

307

by the type of the N-terminal sequence. However, the direction of the shift is not certain. If FRET measured a single donor-acceptor distance between AEDANS bound to TM and DABMI attached to Cys 374 of only one actin subunit, shortening the distance would mean shifting the C-terminal segment towards actin's subdomain 1, where myosin-binding sites are located. This, however would be in discrepancy with the earlier observations that LMW isoforms are better activators of actomyosin ATPase than HMW isoforms (Skorzewski et al., 2009). However, the single TM-bound donor in the TM-actin complex is surrounded by multiple acceptors attached to actin subunits, which contribute to the energy transfer with various efficiencies (Bacchiocchi et al. 2004). According to 3D reconstructions of the filaments' electron micrographs, binding of myosin to actin shifts TM azimuthally towards the inner domain of actin filament (Lehman et al. 1994; Lehman et al. 2000; Xu et al. 1999). Such a shift might increase FRET transfer efficiency between donor and acceptors attached to actin subunits, which belong to the second chain of the long-pitch actin helix. To verify this possibility, we used atomic models of actin-TM (Li et al., 2011) and actin-TM-S1 (Behrman et al., 2012) and analyzed changes in distances between Cys 190 in the central region of TM and the five closest Cys 374 residues. The cysteines were located in three actin subunits, which directly bound $\mathrm{TM}$ along the filament $\left(\mathrm{A}_{-1}, \mathrm{~A}_{0}\right.$ and $\left.\mathrm{A}_{+1}\right)$, and in two subunits across the filament ( $\mathrm{A}_{-2}$ and $\left.\mathrm{A}_{22}\right)$. The analysis showed that in the absence of $\mathrm{S} 1$ Cys 374 in $\mathrm{A}_{0}, \mathrm{~A}_{-1}$ and $\mathrm{A}_{-2}$ were the closest to Cys190. In the presence of S1 Cys190 was shifted away from Cys 374 in $\mathrm{A}_{0}$ and $\mathrm{A}_{-1}$, whereas it was moved towards Cys 374 in $\mathrm{A}_{+2}$ and $\mathrm{A}_{-2}$. Thus, in the S1-induced open state the acceptors bound to actin subunits across the filament significantly contributed to FRET efficiency. Even though the ends are missing in the actin-TM-S1 model, and in the actin-TM model the overlap is not resolved, we assume that these considereations also hold true for the end-to-end junction. Binding of S1 diminished the FRET distances of LMW 
isoforms, and therefore it appears that, in these isoforms, the end-to-end overlap was more to actin shifts TM by as much as $23 \AA$. The $\mathrm{S} 1$-induced shift which we observed in this and the previous work was much shorter, which could be explained by the multi-acceptor system

313 discussed above as well as by local differences in TM isoforms bending. C-termini of HMW and LMW isoforms were shifted respectively further or closer totransfer.

Our data has also shown very high cooperativity of the S1-induced activation of the filament. Depending on the isoform, the maximal shift was completed when about 1-2 myosin heads per one TM molecule was bound.

325 It is worth noting that the C-terminal segment of TM1b9a showed similar cooperativity as the C-termini of the 326 other isoforms. However, it was much larger than the cooperativity of the N-terminal segment of TM1b9a, which required about 0.4 S1/actin for half maximal saturation of the changes in FRET distance (Sliwinska et al. 2011). This result supports our conclusion that both ends of this chimeric TM are not compatible with each other.

\section{Conclusions}

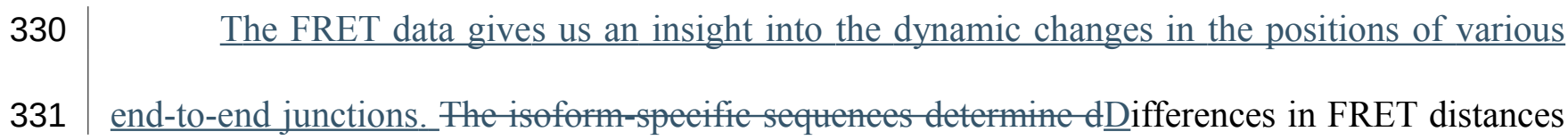


332

333

334

335

336

337

338

339

340

341 mechanisms of the thin filament regulation.

\section{Acknowledgements}

\section{References}

Bacchiocchi C, Graceffa P, and Lehrer SS. 2004. Myosin-induced movement of alphaalpha,
We thank William Lehman and Edward Li for sharing with us the coordinates of the actin-TM

$\underline{\text { model. }}$

The authors thank Katarzyna Robaszkiewiez and Hreneusz Moraezewski for their help with

preparation of Figures 1 and 4. 
351 Barua B, Fagnant PM, Winkelmann DA, Trybus KM, and Hitchcock-Degregori SE. 2013. A

352

353

354

355

356

357

358

359

360

361

362

363

364

365

366

367

368

369

370

371

372

373

374

375 periodic pattern of evolutionarily-conserved basic and acidic residues constitutes the binding interface of actin- tropomyosin. Journal of Biological Chemistry 288: 9602-9609.

Barua B, Pamula MC, and Hitchcock-DeGregori SE. 2011. Evolutionarily conserved surface residues constitute actin binding sites of tropomyosin. Proceedings of the National Academy of Sciences of the United States of America 108:10150-10155.

Barua B, Winkelmann DA, White HD, and Hitchcock-DeGregori SE. 2012. Regulation of actinmyosin interaction by conserved periodic sites of tropomyosin. Proceedings of the National Academy of Sciences of the United States of America 109:18425-18430.

Behrmann E, Muller M, Penczek PA, Mannherz HG, Manstein DJ, and Raunser S. 2012. Structure of the rigor actin-tropomyosin-myosin complex. Cell 150:327-338.

Eaton BL. 1976. Tropomyosin binding to F-actin induced by myosin heads. Science 192:13371339.

Frye J, Klenchin VA, and Rayment I. 2010. Structure of the tropomyosin overlap complex from chicken smooth muscle: insight into the diversity of N-terminal recognition. Biochemistry 49:4908-4920.

Greenfield NJ, Huang YJ, Swapna GV, Bhattacharya A, Rapp B, Singh A, Montelione GT, and Hitchcock-DeGregori SE. 2006. Solution NMR structure of the junction between tropomyosin molecules: implications for actin binding and regulation. Journal of Molecular Biology 364:80-96.

Greenfield NJ, Kotlyanskaya L, and Hitchcock-DeGregori SE. 2009. Structure of the N terminus of a nonmuscle alpha-tropomyosin in complex with the $\mathrm{C}$ terminus: implications for actin binding. Biochemistry 48:1272-1283.

Greenfield NJ, Swapna GV, Huang Y, Palm T, Graboski S, Montelione GT, and HiteheockĐeGregeri SE. 2003. The structure of the earboxyl terminus of striated alpha-tropemyosin 
378 | Gunning P, O'Neill G, and Hardeman E. 2008. Tropomyosin-based regulation of the actin 379 cytoskeleton in time and space. Physiological Reviews 88:1-35.

380 Holmes KC, and Lehman W. 2008. Gestalt-binding of tropomyosin to actin filaments. Journal of 381 Muscle Research and Cell Motility 29:213-219.

382 Lakowicz J. 1999. Principles of Fluorescence Spectroscopy. New York, Boston, Dordrecht, 383 London, Moscow: Kluwer Academic/Plenum Publishers.

384 Lees-Miller JP, and Helfman DM. 1991. The molecular basis for tropomyosin isoform diversity. 385 Bioessays 13:429-437.

386 Lehman W, and Craig R. 2008. Tropomyosin and the steric mechanism of muscle regulation. 387 Advances in Experimental Medicine and Biology 644:95-109.

388 Lehman W, Craig R, and Vibert P. 1994. Ca(2+)-induced tropomyosin movement in Limulus thin 389 filaments revealed by three-dimensional reconstruction. Nature 368:65-67.

390 Lehman W, Hatch V, Korman V, Rosol M, Thomas L, Maytum R, Geeves MA, Van Eyk JE, 391 Tobacman LS, and Craig R. 2000. Tropomyosin and actin isoforms modulate the 392 localization of tropomyosin strands on actin filaments. Journal of Molecular Biology 393 302:593-606.

394 Lehrer SS, and Morris EP. 1982. Dual effects of tropomyosin and troponin-tropomyosin on 395 actomyosin subfragment 1 ATPase. Journal of Biological Chemistry 257:8073-8080.

Li XE, Tobacman LS, Mun JY, Craig R, Fischer S, and Lehman W. 2011. Tropomyosin position on F-actin revealed by EM reconstruction and computational chemistry. Biophysical Journal 100:1005-1013.

Miki M, Makimura S, Sugahara Y, Yamada R, Bunya M, Saitoh T, and Tobita H. 2012. A three400 dimensional FRET analysis to construct an atomic model of the actin-tropomyosin- 

420:40-55.

403

404

405

406

407

408

409

410

411

412

413

414

415

416

417

418

419

420

421

422

Moraczewska J. 2002. Structural determinants of cooperativity in acto-myosin interactions. Acta Biochimica Polonica 49:805-812.

Moraczewska J, Nicholson-Flynn K, Hitchcock-DeGregori SE. 1999. The ends of tropomyosin are major determinants of actin affinity and myosin subfragment 1 -induced binding to Factin in the open state. Biochemistry. 38:15885-15892.

Murakami K, Stewart M, Nozawa K, Tomii K, Kudou N, Igarashi N, Shirakihara Y, Wakatsuki S, Yasunaga T, and Wakabayashi T. 2008. Structural basis for tropomyosin overlap in thin (actin) filaments and the generation of a molecular swivel by troponin-T. Proceedings of the National Academy of Sciences of the United States of America 105:7200-7205.

Robaszkiewicz K, Dudek E, Kasprzak AA, and Moraczewska J. 2012. Functional effects of congenital myopathy-related mutations in gamma-tropomyosin gene. Biochimica et Biophysica Acta 1822:1562-1569.

Skorzewski R, Sliwinska M, Borys D, Sobieszek A, and Moraczewska J. 2009. Effect of actin Cterminal modification on tropomyosin isoforms binding and thin filament regulation. Biochimica et Biophysica Acta 1794:237-243.

Sliwinska M, Zukowska M, Borys D, and Moraczewska J. 2011. Different positions of tropomyosin isoforms on actin filament are determined by specific sequences of end-toend overlaps. Cytoskeleton (Hoboken) 68:300-312.

White HD. 1982. Special instrumentation and techniques for kinetic studies of contractile systems. Methods in Enzymology 85:698-708. 
423 Xu C, Craig R, Tobacman L, Horowitz R, and Lehman W. 1999. Tropomyosin positions in 424 regulated thin filaments revealed by cryoelectron microscopy. Biophysical Journal 425 77:985-992. 


\section{Figure 1}

Schematic illustration of the types of end-to-end junctions present in TM isoforms used in this work.

C-terminal sequences encoded by exon 9a (green) or 9d (red) overlapped with $\mathrm{N}$-terminal sequences encoded by exons $1 \mathrm{a}$ (orange) or $1 \mathrm{~b}$ (blue). The residues in C-terminal segments, which were mutated to cysteines are shown.

\section{Ala269Cys}

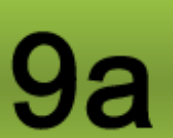

\section{Ser269Cys}
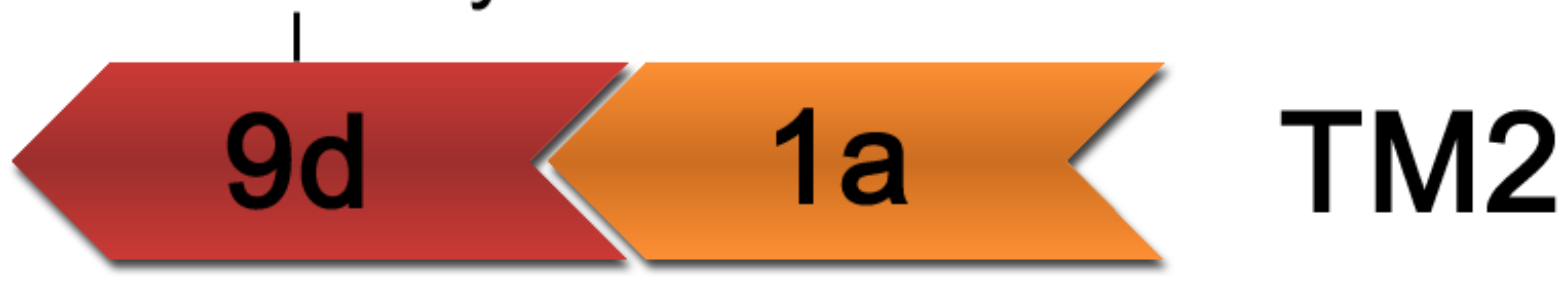

\section{Ala232Cys}

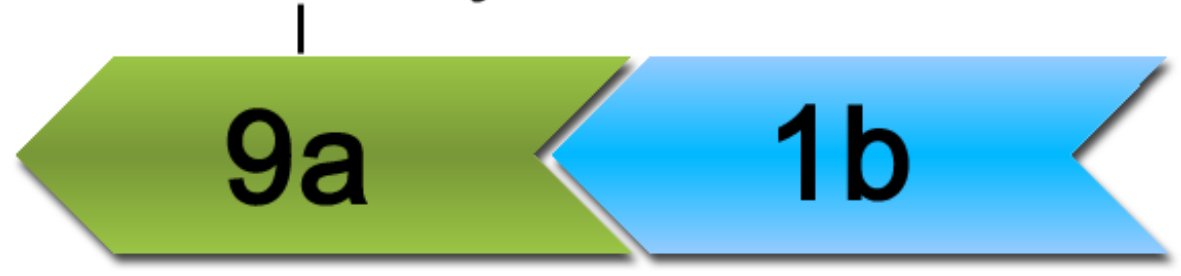

TM1b9a

\section{Ser232Cys}
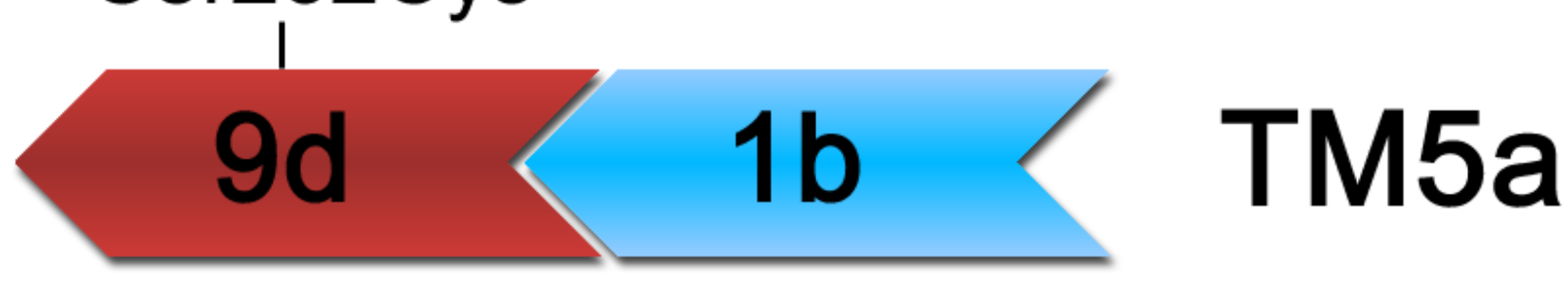


\section{Figure 2}

Quenching of AEDANS-TM fluorescence by DABMI-actin.

sk TM (closed circles, solid line), TM2 (open circles, long dash), TM5a (open squares, short dash) and TM1b9a (closed squares, dotted line) at $0.6 \mu \mathrm{M}$ were titrated with DABMI-actin. The data was normalized using equation: $\left(I-I_{\min }\right) /\left(I_{\max }{ }^{-I} \min \right)$, where $I_{\max }$ is the minimal intensity of the fluorescence of AEDANS-TM alone and $I_{\min }$ is the minimal fluorescence intensity obtained in the presence of DABMI-actin. The lines were generated by fitting the experimental points to ligandbinding equation in Sigma Plot. Conditions: $2 \mathrm{mM} \mathrm{HEPES,} \mathrm{pH} \mathrm{7.6,} 40 \mathrm{mM} \mathrm{NaCl}, 5 \mathrm{mM} \mathrm{MgCl}_{2}$ at $22{ }^{\circ} \mathrm{C}$. Excitation and emission wavelengths were $340 \mathrm{~nm}$ and $495 \mathrm{~nm}$, respectively. The points were averaged from 3 to 5 independent experiments.

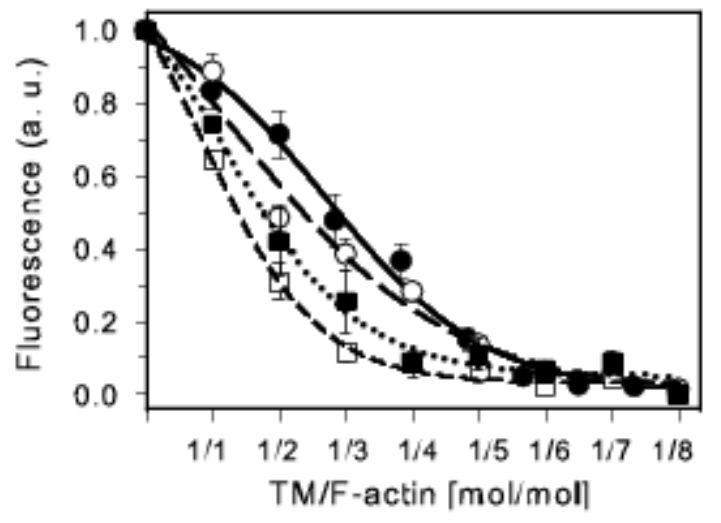




\section{Figure 3}

Myosin S1-induced changes in fluorescence of AEDANS-TM bound to DABMI-actin.

(A) HMW TM isoforms: skTM (closed circles, solid line), TM2 (open circles, long dash line) (B) LMW TM isoforms: TM5a (open squares, short dash line), TM1b9a (closed squares, dotted line). AEDANS-TM isoforms at $0.6 \mu \mathrm{M}$ were bound to $4.8 \mu \mathrm{M}$ DABMI-actin and titrated with myosin S1. The data obtained for $\mathrm{HMW}$ isoforms was normalized by using the equation $\left(\mathrm{I}-\mathrm{I}_{\mathrm{A}}\right) /\left(\mathrm{I}_{\mathrm{S} 1}{ }^{-\mathrm{I}} \mathrm{A}\right)$. For LMW isoforms the equation was: $\left(\mathrm{II}_{\mathrm{S}_{1}}\right) /\left(\mathrm{I}_{\mathrm{A}}{ }^{-I} \mathrm{~S}_{1}\right)$, where I was the fluorescence intensity at given titration point; I ${ }_{A}$ was the intensity of TM-F-actin complex in the absence of $S 1$; I $S_{1}$ was the fluorescence intensity of AEDANS-TM at maximal S1 concentration. The points were averaged from 3 to 4 independent experiments. The lines were obtained by fitting the experimental data to the Hill equation. Conditions as described under Table 3.
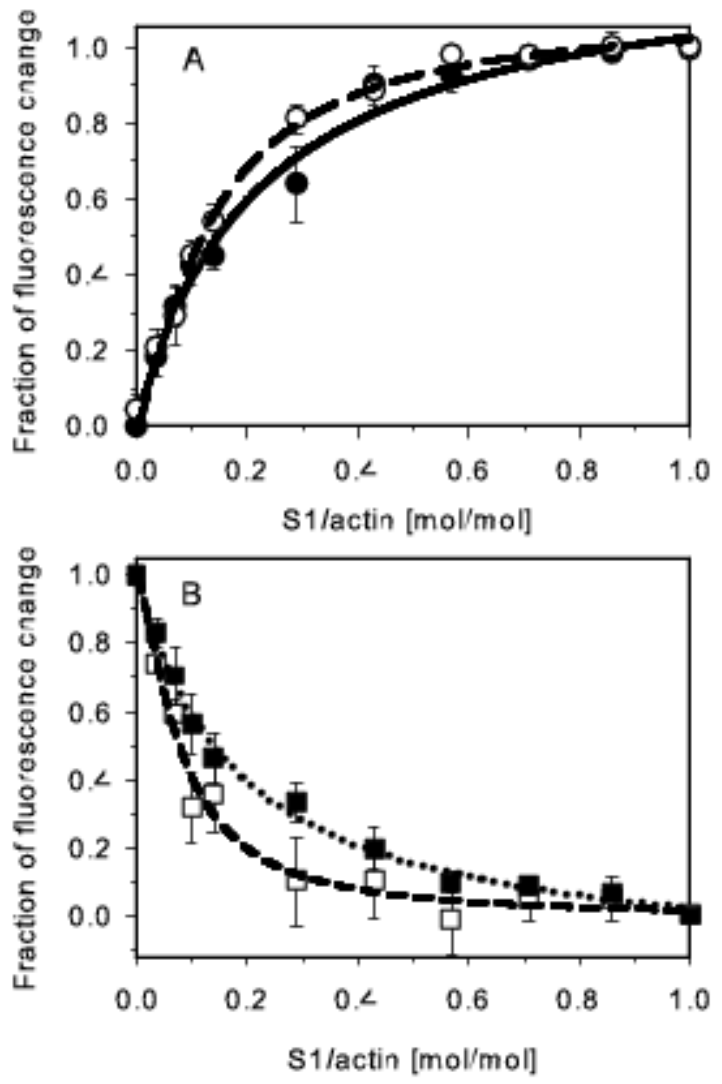


\section{Table 1 (on next page)}

Localization of cysteine mutation sites in C-termini encoded by exons 9a and 9d

The upper row shows positions of the amino acid residues in the coiled-coil heptapeptide repeat. Ala or Ser residues changed into Cys to create AEDANS attachment sites are in red. 
1

\begin{tabular}{lllllllllllllllllllllllllllll}
\hline & $f$ & $g$ & $a$ & $b$ & $c$ & $d$ & $e$ & $f$ & $g$ & $a$ & $b$ & $c$ & $d$ & $e$ & $f$ & $g$ & $a$ & $b$ & $c$ & $d$ & $e$ & $f$ & $g$ & $a$ & $b$ & $c$ & $d$ \\
\hline $\mathbf{9}$ & $\mathbf{D}$ & $\mathbf{E}$ & $\mathbf{L}$ & $\mathbf{Y}$ & $\mathbf{A}$ & $\mathbf{Q}$ & $\mathbf{K}$ & $\mathbf{L}$ & $\mathbf{K}$ & $\mathbf{Y}$ & $\mathbf{K}$ & $\mathbf{A} /$ & $\mathbf{I}$ & $\mathbf{S}$ & $\mathbf{E}$ & $\mathbf{E}$ & $\mathbf{L}$ & $\mathbf{D}$ & $\mathbf{H}$ & $\mathbf{A}$ & $\mathbf{L}$ & $\mathbf{N}$ & $\mathbf{D}$ & $\mathbf{M}$ & $\mathbf{T}$ & $\mathbf{S}$ & $\mathbf{I}$
\end{tabular}

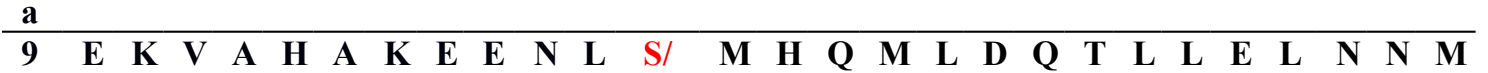
d C

2 


\section{Table 2 (on next page)}

Functional properties of wild type and mutant tropomyosin isoforms labeled with AEDANS

Conditions: $2 \mathrm{mM} \mathrm{HEPES,} \mathrm{pH} \mathrm{7.6,} 40 \mathrm{mM} \mathrm{NaCl}, 5 \mathrm{mM} \mathrm{MgCl}_{2}$ at $22^{\circ} \mathrm{C}$. The numbers are average values \pm S.E. taken from 2 to 9 independent experiments. 


\begin{tabular}{|c|c|c|}
\hline TM isoform & $K_{\text {app }}\left(\times 10^{7} M^{-1}\right)$ & $\begin{array}{c}\text { Actin-myosin S1 ATPase activity } \\
\text { (nmol Pi/mg S1/min.) }\end{array}$ \\
\hline skTM & $1.63 \pm 0.57$ & $103.0 \pm 8.0$ \\
\hline skTM/A269C & $1.80 \pm 0.26$ & $103.8 \pm 7.0$ \\
\hline TM2 & $2.25 \pm 0.76$ & $91.7 \pm 13.0$ \\
\hline TM2/S269C & $2.04 \pm 0.45$ & $112.0 \pm 10.0$ \\
\hline TM5a & $1.42 \pm 0.36$ & $169.0 \pm 16.0$ \\
\hline TM5a/S232C & $1.49 \pm 0.58$ & $183.8 \pm 20.0$ \\
\hline TM1b9a & $1.27 \pm 0.40$ & $108.2 \pm 9.0$ \\
\hline TM1b9a/A232C & $1.24 \pm 0.44$ & $125.5 \pm 9.0$ \\
\hline
\end{tabular}

2 


\section{Table 3 (on next page)}

Anisotropy of AEDANS bound to C-terminal cysteine residues of TM isoforms

Conditions: $0.6 \mu \mathrm{M}$ AEDANS-labeled TM alone and with $4.8 \mu \mathrm{M}$ actin $\pm 5 \mu \mathrm{M}$ S1 in $2 \mathrm{mM}$ HEPES, pH 7.6, $40 \mathrm{mM} \mathrm{NaCl}, 5 \mathrm{mM} \mathrm{MgCl}_{2}$ at $22^{\circ} \mathrm{C}$. Excitation and emission wavelength were $340 \mathrm{~nm}$ and $495 \mathrm{~nm}$, respectively. Average values \pm S.E. were taken from 3 to 8 independent measurements. 
1

\begin{tabular}{llll}
\hline TM isoform & TM alone & TM-actin & TM-actin-S1 \\
\hline skTM/A269C & $0.073 \pm 0.008$ & $0.086 \pm 0.008$ & $0.123 \pm 0.012$ \\
TM2/S269C & $0.072 \pm 0.002$ & $0.106 \pm 0.005$ & $0.137 \pm 0.004$ \\
TM5a/S232C & $0.066 \pm 0.003$ & $0.096 \pm 0.004$ & $0.124 \pm 0.004$ \\
TM1b9a/A232C & $0.095 \pm 0.008$ & $0.104 \pm 0.003$ & $0.127 \pm 0.009$ \\
\hline
\end{tabular}

2 


\section{Table 4 (on next page)}

Spectral parameters of FRET between AEDANS-labeled tropomyosins and DABMIlabeled actin

Conditions as given under Table 3. Average values \pm S.E. were taken from 10 to 14 independent experiments. 
1

\begin{tabular}{lccccc}
\hline TM isoform & $\mathbf{Q}_{\mathbf{D}}$ & $\begin{array}{c}\mathbf{J}\left(\mathbf{x 1 0}^{\mathbf{1 4}} \mathbf{n m}^{\mathbf{4}}\right. \\
\mathbf{M}^{\mathbf{1}}{\mathbf{~} \mathbf{m}^{-\mathbf{1}}}^{\mathbf{n}}\end{array}$ & $\mathbf{R}_{\mathbf{0}}(\AA)$ & $\mathbf{E}$ & $\mathbf{R}(\AA)$ \\
\hline skTM/A269C & 0.14 & 6.838 & 34.7 & $0.40 \pm 0.01$ & $37.3 \pm 0.21$ \\
TM2/S269C & 0.08 & 6.924 & 32.1 & $0.32 \pm 0.01$ & $36.4 \pm 0.21$ \\
TM5a/S232C & 0.20 & 7.012 & 37.1 & $0.32 \pm 0.03$ & $42.7 \pm 1.20$ \\
TM1b9a/A232 & 0.12 & 7.087 & 34.3 & $0.13 \pm 0.02$ & $48.1 \pm 1.45$ \\
C & & & & & \\
\hline
\end{tabular}

2

3 


\section{Table 5 (on next page)}

S1-induced changes in FRET between AEDANS-labeled TMs and DABMI-labeled actin

Conditions as given under Table 3. Average values \pm S.E. were taken from 6 to 9 independent experiments. S1/actin molar ratio is the ratio of strongly bound myosin heads required for half-maximal change in FRET. In parentheses, S1/TM molar ratio obtained by multiplying S1/actin by the number of actin subunits bound by one molecule of each TM isoform. 


\begin{tabular}{lcccccc}
\hline TM isoform & $\mathbf{Q}_{\mathbf{D}}$ & $\mathbf{R}_{\mathbf{0}}$ & $\mathbf{E}$ & $\mathbf{R}(\AA)$ & $\begin{array}{c}\Delta \mathbf{R}_{\mathbf{S 1}} \\
(\AA)\end{array}$ & $\begin{array}{c}\text { S1/actin molar } \\
\text { ratio }\end{array}$ \\
\hline skTM/A269C & 0.15 & 35.0 & $0.28 \pm 0.01$ & $41.0 \pm 0.1$ & 3.7 & $0.15 \pm 0.02(1.05)$ \\
TM2/S269C & 0.09 & 32.5 & $0.26 \pm 0.02$ & $38.8 \pm 0.2$ & 2.4 & $0.12 \pm 0.01(0.84)$ \\
TM5a/S232C & 0.22 & 37.6 & $0.44 \pm 0.02$ & $38.9 \pm 0.4$ & -3.8 & $0.08 \pm 0.02(0.48)$ \\
TM1b9a/A232C & 0.14 & 35.0 & $0.24 \pm 0.03$ & $42.6 \pm 1.2$ & -5.5 & $0.14 \pm 0.03(0.84)$ \\
\hline
\end{tabular}

